PUPT-1685

Imperial/TP/96-97/33

hep-th/9703040

\title{
String Theory and Classical Absorption by Threebranes
}

\author{
Steven S. Gubser, ${ }^{1}$ Igor R. Klebanov, ${ }^{2}$ \\ Joseph Henry Laboratories, Princeton University, Princeton, NJ 08544 \\ and \\ Arkady A. Tseytlin ${ }^{3 \dagger}$ \\ Blackett Laboratory, Imperial College, London SW7 2BZ, U.K.
}

\begin{abstract}
Low energy absorption cross-sections for various particles falling into extreme nondilatonic branes are calculated using string theory and world-volume field theory methods. The results are compared with classical absorption by the corresponding gravitational backgrounds. For the self-dual threebrane, earlier work by one of us demonstrated precise agreement of the absorption cross-sections for the dilaton, and here we extend the result to Ramond-Ramond scalars and to gravitons polarized parallel to the brane. In string theory, the only absorption channel available to dilatons and Ramond-Ramond scalars at leading order is conversion into a pair of gauge bosons on the threebrane. For gravitons polarized parallel to the brane, scalars, fermions and gauge bosons all make leading order contributions to the cross-section, which remarkably add up to the value predicted by classical gravity. For the twobrane and fivebrane of M-theory, numerical coefficients fail to agree, signalling our lack of a precise understanding of the world-volume theory for large numbers of coincident branes. In many cases, we note a remarkable isotropy in the final state particle flux within the brane. We also consider the generalization to higher partial waves of minimally coupled scalars. We demonstrate agreement for the threebrane at $\ell=1$ and indicate that further work is necessary to understand $\ell>1$.
\end{abstract}

March 1997

\footnotetext{
1 e-mail: ssgubser@viper.princeton.edu

2 e-mail: klebanov@viper.princeton.edu

3 e-mail: tseytlin@ic.ac.uk

$\dagger$ Also at Lebedev Physics Institute, Moscow.
} 


\section{Introduction}

Dirichlet branes provide an elegant embedding of Ramond-Ramond charged objects into string theory [1]. The D-brane description of the dynamics of these solitons may be compared with corresponding results in the semi-classical low-energy supergravity. In particular, a counting of degeneracies for certain intersecting D-branes reproduces, in the limit of large charges, the Bekenstein-Hawking entropy of the corresponding geometries [2, 3]. Furthermore, calculations of emission and absorption rates for scalar particles agree with a simple 'effective string' model for the dynamics of the intersection [3, 4, 5, 6, 0, 8, 8, 9, 10 . There are some signs, however, that the simplest model is not capable of incorporating all the complexities of black holes physics [11, 12, 13]. This is probably due to the fact that the dynamics of intersecting D-branes, which is not yet fully understood, cannot be captured by one simple model.

Another line of development, which has proceeded in parallel to the investigations of intersecting D-branes, concerns the simpler configurations which involve parallel D-branes only. Their string theoretic description is well understood in terms of supersymmetric $U(N)$ gauge theory on the world-volume [14]. Pertrubative string calculations of scattering [15,16] and absorption [17] are fairly straightforward for the parallel D-branes, and their low-energy dynamics is summarized in the DBI action.

To leading order in the string coupling, $N$ coincident Dp-branes are described by $O\left(N^{2}\right)$ free fields in $p+1$ dimensions. This result may be compared with the BekensteinHawking entropy of the near-extremal $p$-brane solutions in supergravity. In [18, 19] it was found the the scaling of the Bekenstein-Hawking entropy with the temperature agrees with that for a massless gas in $p$ dimensions only for the 'non-dilatonic $p$-branes'. The only representative of this class which is described by parallel D-branes is the self-dual threebrane. Other representatives include the dyonic string in $D=6$, and the twobranes and fivebranes of M-theory. In [20] a way of reconciling the differing scalings for the dilatonic branes [21] was proposed.1] Neveretheless, in [23] it was shown that the non-dilatonic branes (and especially the self-dual threebrane) have a number of special properties that allow for more detailed comparisons between semi-classical gravity and the microscopic theory. For example, the string theoretic calculation of the absorption cross-section by threebranes for low-energy dilatons was found to agree exactly with the classical calculation in the background of the extremal classical geometry [23].

What are the features that make the threebrane so special? In classical supergravity, the extremal threebrane is the only RR-charged solution that is perfectly non-singular [24,25]. For $N$ threebranes, the curvature of the classical solution is bounded by a quantity of order

$$
\frac{1}{\sqrt{N \kappa_{10}}} \sim \frac{1}{\alpha^{\prime} \sqrt{N g_{\mathrm{str}}}}
$$

1 Other ideas on how to find agreement for the dilatonic branes were put forward in [22]. 
Thus, to suppress the string scale corrections to the classical metric, we need to take the limit $N g_{\text {str }} \rightarrow \infty$. This fact seems to lead to a strongly coupled theory on the world-volume and raises questions about the applicability of string perturbation theory to macroscopic threebranes. However, in [23] it was shown that the dimensionless expansion parameter that enters the string theoretic calculation of the absorption cross-section is actually

$$
N \kappa_{10} \omega^{4} \sim N g_{\mathrm{str}} \alpha^{\prime 2} \omega^{4}
$$

where $\omega$ is the incident energy. Thus, we may consider a 'double scaling limit,'

$$
N g_{\mathrm{str}} \rightarrow \infty, \quad \omega^{2} \alpha^{\prime} \rightarrow 0
$$

where the expansion parameter (1.1) is kept small. Moreover, the classical absorption cross-

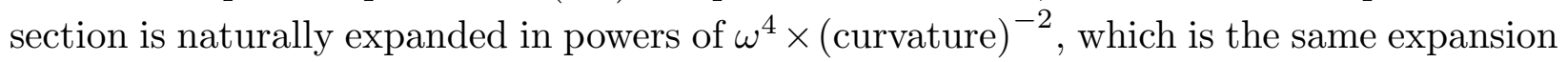
parameter (1.1) as the one governing the string theoretic description of the threebranes. The two expansions of the cross-section thus may indeed be compared, and the leading term agrees exactly [23]. This provides strong evidence in favor of absorption by extremal threebranes being a unitary process. While in the classical calculation the information carried by the dilaton seems to disappear down the infinite throat of the classical solution, the stringy approach indicates that the information is not lost: it is stored in the quantum state of the back-to-back massless gauge bosons on the world-volume which are produced by the dilaton. Subsequent decay of the threebrane back to the ground state proceeds via annihilation of the gauge bosons into an outgoing massless state, and there seems to be no space for information loss.

The scenario mentioned above certainly deserves a more careful scrutiny. In this paper we carry out further comparisons between string theory and classical gravity of the threebrane. In string theory there is a variety of fields that act as minimally coupled massless scalars with respect to the transverse dimensions. The dilaton, which was investigated in [23], is perhaps the simplest one to study. In this paper we turn to other such fields: the RR scalar and the gravitons polarized parallel to the world-volume. We show that in classical gravity all these fields satisfy the same equation and, therefore, are absorbed with the same rate as the dilaton. However, their cubic couplings to the massless worldvolume modes dictated by string theory [17] are completely different. In particular, the longitudinally polarized gravitons can turn into pairs of gauge bosons, scalars, or fermions. Adding up these rates we find that the total cross-section has the same universal value, in agreement with classical gravity.

Another interesting check concerns the absorption of scalars in partial waves higher than $\ell=0$. In [23] it was shown that the scaling of the relevant cross-sections with $\omega$ and $N$ agrees for all $\ell$. For $\ell=1$ we show that the coefficient agrees as well. For $\ell>1$ the simplest assumption about the effective action does not yield a coefficient which agrees 
with the classical calculation. Normalization of the effective action is a subtle matter, however, and we suspect that its direct determination from string amplitudes will yield agreement with the classical cross-sections.

While a microscopic description of RR-charged branes is by now well known in string theory, the situation is not as simple for the p-brane solutions of the 11-dimensional supergravity. There is good evidence that a yet unknown M-theory underlies their fundamental description. Comparisons with semi-classical gravity provide consistency checks on this description. In [23] the scalings of the classical absorption cross-sections were found to agree with the twobrane and fivebrane effective action considerations. Here we calculate the absorption cross-section of a longitudinally polarized graviton by a single fivebrane. Using the world-volume effective action, we find that the absorption cross-section is $1 / 4$ of the rate formally predicted by classical gravity. In fact, one could hardly expect perfect agreement for a single fivebrane - the classical description is expected to be valid only for a large number of coincident branes. It is interesting, nevertheless, how close the two calculations come to agreeing with each other. We also carry out a similar comparison for a single twobrane, but find the discrepancy in the coefficient to be far greater than in the fivebrane case.

The structure of the paper is as follows. In section 2 we exhibit the space-time effective actions and derive the classical equations satisfied by various fields. In section 3 we present the new threebrane calculations. In section 4 we carry out the comparisons of classical 11-dimensional supergravity with the predictions of the M-brane world-volume actions. We conclude in section 5 .

\section{Space-time effective actions and perturbations around non-dilatonic p-brane solutions}

\subsection{Type IIB theory perturbations near threebranes}

The bosonic part of the field equations of $d=10$ type IIB supergravity, which is the low-energy limit of the type IIB superstring [26], can be derived from the following action: 27,28

$$
\begin{gathered}
S_{10}=\frac{1}{2 \kappa_{10}^{2}} \int d^{10} x\left[\sqrt { - G } \left(e^{-2 \phi}\left[R+4(\partial \phi)^{2}-\frac{1}{12}\left(\partial B_{2}\right)^{2}\right]\right.\right. \\
\left.\left.-\frac{1}{2}(\partial C)^{2}-\frac{1}{12}\left(\partial C_{2}-C \partial B_{2}\right)^{2}-\frac{1}{4 \cdot 5 !} F_{5}^{2}\right)-\frac{1}{2 \cdot 4 ! \cdot(3 !)^{2}} \epsilon_{10} C_{4} \partial C_{2} \partial B_{2}+\ldots\right]
\end{gathered}
$$

where 2

$$
\left(\partial B_{2}\right)_{M N K} \equiv 3 \partial_{[M} B_{N K]}, \quad\left(\partial C_{4}\right)_{M N K L P} \equiv 5 \partial_{[M} C_{N K L P]}
$$

2 We use the following notation. The signature is $(-+\ldots+) . M, N, \ldots$ label the coordinate indices of $d=10$ or $d=11$ theory. The indices $\alpha, \beta, \ldots(a, b, .$.$) will label the space-time (spatial)$ 


$$
F_{5}=\partial C_{4}+5\left(B_{2} \partial C_{2}-C_{2} \partial B_{2}\right) .
$$

Following [28] we assume that the self-duality constraint $F_{5}=\tilde{F}_{5}$ may be added at the level of the equations of motion. This action is a useful tool for deriving the dimensionally reduced forms of type IIB supergravity action [28] and also for discussing perturbations near the solitonic p-brane solutions given below. Written in the Einstein frame $\left(g_{M N}=\right.$ $\left.e^{-\phi / 2} G_{M N}\right)$ it takes the following $S L(2, R)$ covariant form:

$$
\begin{gathered}
S_{10}=\frac{1}{2 \kappa_{10}^{2}} \int d^{10} x\left(\sqrt { - g _ { 1 0 } } \left[R-\frac{1}{2}(\partial \phi)^{2}-\frac{1}{12} e^{-\phi}\left(\partial B_{2}\right)^{2}\right.\right. \\
\left.\left.-\frac{1}{2} e^{2 \phi}(\partial C)^{2}-\frac{1}{12} e^{\phi}\left(\partial C_{2}-C \partial B_{2}\right)^{2}-\frac{1}{4 \cdot 5 !} F_{5}^{2}\right]-\frac{1}{2 \cdot 4 ! \cdot(3 !)^{2}} \epsilon_{10} C_{4} \partial C_{2} \partial B_{2}+\ldots\right) .
\end{gathered}
$$

The extremal 3-brane of type IIB theory is represented by the background 29,24

$$
\begin{gathered}
d s_{10}^{2}=H^{-1 / 2}\left(-d t^{2}+d x_{a} d x_{a}\right)+H^{1 / 2} d x_{i} d x_{i} \\
\left(\partial C_{4}\right)_{a b c d i}=\epsilon_{a b c d} \partial_{i} H^{-1}, \quad\left(\partial C_{4}\right)_{i j k l m}=\epsilon_{i j k l m n} \partial_{n} H, \quad H=1+\frac{R^{4}}{r^{4}}, \quad r^{2}=x_{i} x_{i}
\end{gathered}
$$

with all other components and fields vanishing (i.e. $\phi=C=B_{2}=C_{2}=0, \quad F_{5}=\partial C_{4}$ ). The scalar curvature $\sim\left(\partial C_{4}\right)^{2} \sim R^{-2}$ near $r=0$, In fact, this metric may be extended to a geodesically complete non-singular geometry [25]. This is the only RR-charged p-brane for which this is possible.

Let us consider small perturbations near this background which depend only on $x_{\mu}$, i.e. on the time $t$ and the transverse coordinates $x_{i}$. Our aim is to identify the modes which have simple Klein-Gordon type equations. A guiding principle is to look for (components of) the fields that have trivial background values.

The obvious examples are the dilaton $\phi$ and the Ramond-Ramond scalar $C$ perturbations which are decoupled from the $C_{4}$-background and thus have the action $(\varphi=(\phi, C))$

$$
S_{\text {scal. }}=-\frac{1}{4 \kappa_{10}^{2}} \int d^{10} x \sqrt{-g_{10}} g^{\mu \nu} \partial_{\mu} \varphi \partial_{\nu} \varphi=-\frac{1}{4 \kappa_{10}^{2}} \int d^{10} x\left[\partial_{i} \varphi \partial_{i} \varphi-H(r) \partial_{0} \varphi \partial_{0} \varphi\right]
$$

where we have used the fact that for the 3-brane background the Einstein and the string frame metrics are identical, $G_{M N}=g_{M N}$, which implies $g_{\mu \nu}=\operatorname{diag}\left(-H^{-1 / 2}, H^{1 / 2} \delta_{i j}\right)$, $\sqrt{-g_{10}}=H^{1 / 2}$.

coordinates parallel to the p-brane world-volume, i.e. $\alpha=(0, a), a=1, \ldots, p$; the indices $i, j, \ldots$, will label the coordinates transverse to the p-brane, $i=p+1, \ldots, 9(10)$. We shall also use $\mu, \nu, \ldots$ for the indices of the dimensionally reduced theory obtained by compactifying the internal directions parallel to the p-brane, i.e. $\mu=(0, i) . \epsilon_{d}$ will stand for the totally antisymmetric symbol (density). Contractions over repeated lower-case indices are always performed with the flat metric, and $A_{[a b]} \equiv \frac{1}{2}\left(A_{a b}-A_{b a}\right)$. 
Perturbations of the metric are, in general, mixed with perturbations of components of $C_{4}$. An important exception is the traceless part of the longitudinal (polarized along the 3-brane) graviton perturbations $h_{a b}$. These perturbations give rise to scalars upon dimensional reduction to $d=7$. Let us split the metric in the ' $7+3$ ' fashion

$$
d s_{10 E}^{2}=g_{\mu \nu} d x^{\mu} d x^{\nu}+g_{a b} d x^{a} d x^{b}
$$

and assume that all the fields depend only on $x^{\mu}$ (this is equivalent to reduction to 7 dimensions). Then the relevant part of (2.2) becomes

$$
S_{10}=\frac{1}{2 \kappa_{10}^{2}} \int d^{10} x \sqrt{-g_{7}} \sqrt{g_{3}}\left(R_{7}-\frac{1}{4} g^{a b} g^{c d} \partial_{\mu} g_{a c} \partial^{\mu} g_{b d}-\frac{1}{48} F_{\mu \nu a b c} F_{d e f}^{\mu \nu} g^{a d} g^{b e} g^{c f}+\ldots\right),
$$

where $g_{7} \equiv \operatorname{det} g_{\mu \nu}, g_{3} \equiv \operatorname{det} g_{a b}$ and we have written down explicitly the only $F_{5}^{2}$ term that could potentially couple $h_{a b}$ to the gauge field strength background. Introducing the 'normalized' metric $\gamma_{a b}=g_{3}^{-1 / 3} g_{a b}$ which has unit determinant, we find

$$
\begin{aligned}
S_{\text {scal.grav. }} & =\frac{1}{2 \kappa_{10}^{2}} \int d^{10} x \sqrt{-g_{7}} \sqrt{g_{3}}\left(R_{7}-\frac{1}{4} \gamma^{a b} \gamma^{c d} \partial_{\mu} \gamma_{a c} \partial^{\mu} \gamma_{b d}\right. \\
& \left.-\frac{1}{12} g_{3}^{-2} \partial_{\mu} g_{3} \partial^{\mu} g_{3}-\frac{1}{8} g_{3}^{-1} F_{\mu \nu} F^{\mu \nu}+\ldots\right)
\end{aligned}
$$

where $F_{\mu \nu}=F_{\mu \nu 123}$ is the $d=7$ vector field strength which, according to (2.4), describes an electrically charged extremal black hole. Thus, only the determinant of $g_{a b}$, which is related to the 7-dimensional dilaton, couples to the gauge field background. This is consistent with the fact that $\gamma_{a b}$ has a trivial background value, $\gamma_{a b}=\delta_{a b}$, while the value of det $g_{a b}$ in $(2.3)$ is $g_{3}=H^{-3 / 2}$.

The fluctuations $h_{a b}=\gamma_{a b}-\delta_{a b}$ (which are traceless, $h_{a a}=0$, to keep det $\gamma_{a b}=1$ ) thus have the same quadratic part of the action as the scalar fields in (2.5)

$$
\begin{gathered}
S_{\text {scal.grav. }}=-\frac{1}{8 \kappa_{10}^{2}} \int d^{10} x \sqrt{-g_{10}} \partial_{\mu} h_{a b} \partial^{\mu} h_{a b} \\
=-\frac{1}{8 \kappa_{10}^{2}} \int d^{10} x\left[\partial_{i} h_{a b} \partial_{i} h_{a b}-H(r) \partial_{0} h_{a b} \partial_{0} h_{a b}\right] .
\end{gathered}
$$

Similar conclusions hold for other p-brane solutions because the 'normalized' internal space metric $\gamma_{a b}$ has a flat background value.

The problem of discussing perturbations that have higher spin from the point of view of the $d=7$ black hole is more difficult because of mixing among different fields. We include some observations on this problem in Appendix. 


\section{2. $d=11$ supergravity perturbations near twobranes and fivebranes}

The discussion of perturbations around the twobrane and fivebrane solutions [30,31] of the $d=11$ supergravity is very similar to the analysis in the preceding subsection. The starting point is the bosonic part of the $d=11$ supergravity action [32]

$$
S_{11}=\frac{1}{2 \kappa_{11}^{2}} \int d^{11} x\left(\sqrt{-g_{11}}\left[R-\frac{1}{2 \cdot 4 !}\left(\partial C_{3}\right)^{2}\right]+\frac{1}{(12)^{4}} \epsilon_{11} C_{3} \partial C_{3} \partial C_{3}+\ldots\right)
$$

where $\left(\partial C_{3}\right)_{M N K L}=4 \partial_{[M} C_{N K L]}$. The twobrane and fivebrane backgrounds are respectively $(a=1, \ldots, p, i=p+1, \ldots, 10, p=2,5)$

$$
\begin{gathered}
d s_{11}^{2}=H^{-2 / 3}\left(-d t^{2}+d x_{a} d x_{a}\right)+H^{1 / 3} d x_{i} d x_{i}, \\
\left(\partial C_{3}\right)_{0 a b n}=\epsilon_{a b} \partial_{n} H^{-1}, \quad H=1+\frac{R^{6}}{r^{6}}, \\
d s_{11}^{2}=H^{-1 / 3}\left(-d t^{2}+d x_{a} d x_{a}\right)+H^{2 / 3} d x_{i} d x_{i}, \\
\left(\partial C_{3}\right)_{i j k l}=\epsilon_{i j k l n} \partial_{n} H, \quad H=1+\frac{R^{3}}{r^{3}} .
\end{gathered}
$$

Both for the twobrane and for the fivebrane, the scalar curvature is $\sim\left(\partial C_{3}\right)^{2} \sim R^{-2}$ near $r=0$. There is a subtle difference, however, in that the fivebrane metric may be extended to a geodesically complete non-singular geometry, while the twobrane metric cannot [25].

We consider small perturbations near these backgrounds which depend only on $x_{\mu}=$ $\left(t, x_{i}\right)$. There are no scalars like $\phi$ or $C$ in the $d=11$ theory, but it is easy to check that, as in (2.6), (2.7), the 'normalized' internal part of the metric $\gamma_{a b}=g_{p}^{-1 / p} g_{a b}, g_{p} \equiv \operatorname{det} g_{a b}$ $(p=2,5)$, which has a flat background value, is decoupled from the $\left(\partial C_{3}\right)^{2}$ term. As a result, the gravitons polarized parallel to the brane, $h_{a b}=\gamma_{a b}-\delta_{a b}, h_{a a}=0$, have the minimal action as in (2.8),

$$
S_{\text {scal.grav. }}=-\frac{1}{8 \kappa_{11}^{2}} \int d^{11} x\left[\partial_{i} h_{a b} \partial_{i} h_{a b}-H(r) \partial_{0} h_{a b} \partial_{0} h_{a b}\right] .
$$

We discuss some other 'mixed' perturbations in Appendix.

\section{The Self-Dual Threebrane}

\subsection{Classical absorption}

This section focuses on minimally coupled scalars, such as those that satisfy the KleinGordon equation following from (2.5), (2.8) with $H(r)$ given in (2.4). Because the classical analysis of absorption for all partial waves of such a scalar was sketched in [23] and is similar to the matching calculations that have appeared in many other places in the literature 
[4, 25, $4,8,9,10,33,34,35,36$, details will not be presented here. In order to employ analytical rather than numerical techniques, it is necessary as usual to take the Compton wavelength much larger than the typical radii of the black hole. Fortunately, it is precisely in this region where agreement with string theoretic models is expected [37].

It is useful to know the Optical Theorem in arbitrary spacetime dimension $d$, which can be derived from properties of the partial wave expansion in arbitrary dimension [38]. The result we need is that the absorption cross-section $\sigma_{\text {abs }}^{\ell}$ of a massless scalar with energy $\omega$ in the $\ell$-th partial wave is related to the absorption probability $1-\left|S_{\ell}\right|^{2}$ by

$$
\sigma_{\mathrm{abs}}^{\ell}=\frac{2^{d-3} \pi^{(d-3) / 2}}{\omega^{d-2}} \Gamma((d-3) / 2)(\ell+(d-3) / 2)\left(\begin{array}{c}
\ell+d-4 \\
\ell
\end{array}\right)\left(1-\left|S_{\ell}\right|^{2}\right)
$$

For the threebrane one takes $d=7$ since the other 3 dimensions are compactified on $T^{3}$. A matching calculation outlined for all partial waves in 23] gives the absorption probability

$$
1-\left|S_{\ell}\right|^{2}=\frac{\pi^{2}(\omega R)^{8+4 \ell}}{[(\ell+1) !]^{4}(\ell+2)^{2} 4^{2 \ell+2}} .
$$

Therefore, the classical result for the cross-section to absorb the $\ell$-th partial wave of a minimally coupled scalar is, to leading order in $(\omega R)^{4}$,

$$
\sigma_{3 \text { class. }}^{\ell}=\frac{\pi^{4}}{24} \frac{(\ell+3)(\ell+1)}{[(\ell+1) !]^{4}}\left(\frac{\omega R}{2}\right)^{4 \ell} \omega^{3} R^{8} .
$$

The scale parameter $R$ of the classical 3-brane solution (2.4) is related [18,23] to the number $N$ of coinciding microscopic 3 -branes by the equation

$$
R^{4}=\frac{\kappa_{10}}{2 \pi^{5 / 2}} N
$$

which follows from the quantization of the threebrane charge.

There is a number of minimally coupled scalars in the theory: the dilaton, the RR scalar, and 'off-diagonal' gravitons polarized with both indices parallel to the 3-brane world-volume. The goal of the next section will be to demonstrate that the universality of leading order cross-section for these scalars, which is so obvious in the semi-classical framework, also follows from the D-brane description.

\subsection{Universality of the absorption cross-section for minimally coupled scalars}

The threebrane is the case where we know the world-volume theory the best: at low energies (and in flat space) it is $\mathcal{N}=4$ supersymmetric $U(N)$ gauge theory where $N$ is the number of parallel threebranes [14]. Thus, the massless fields on the world-volume are the gauge field, 6 scalars, and 4 Majorana fermions, all in the adjoint representation of $U(N)$. 
As we will see below, the universality of the cross-section is not trivial in the world-volume description: while for dilatons and RR scalars leading absorption proceeds by conversion into a pair of gauge bosons only, for the gravitons polarized along the brane it involves a summation over conversions into world-volume scalars, fermions, and gauge bosons.

The world-volume action, excluding all couplings to external fields, is $(I=1, \ldots, 4 ; i=$ $4, \ldots, 9)$

$$
S_{3}=T_{3} \int d^{4} x \operatorname{Tr}\left[-\frac{1}{4} F_{\alpha \beta}^{2}+\frac{i}{2} \bar{\psi}^{I} \gamma^{\alpha} \partial_{\alpha} \psi_{I}-\frac{1}{2}\left(\partial_{\alpha} X^{i}\right)^{2}+\text { interactions }\right] .
$$

As is clear from the $d=10$ origin of this theory, the R-symmetry group is the group $S O(6)$ of spatial rotations in the uncompactified dimensions. Under this group, the gauge fields are neutral and the scalars $X^{i}$ form a 6 . When the fermions are written in chiral components, $\psi_{I}=\left(\begin{array}{l}\lambda_{I} \\ \bar{\lambda}^{I}\end{array}\right)$, the fields $\lambda_{I}$ form a 4 of $S O(6)=S U(4)$.

In order to properly normalize the amplitudes, one needs the kinetic part of the action for the bulk fields, which is given by (2.5) and (2.8).

We also need to know how the threebrane world-volume fields couple to the bulk fields of type IIB supergravity. For a single threebrane, a $\kappa$-symmetric version of the DBI action in a non-trivial type IIB background was constructed in [39] (see also [40]), which, by use of superfields, captures all such couplings to leading order in derivatives of external fields. For the terms necessary to us, the generalization from $U(1)$ to $U(N)$ gauge group is straightforward. One can, in principle, obtain detailed information about the structure of the non-abelian action by directly computing string amplitudes as done in [17]. The threebrane is well suited to this line of attack because the string theory description is known exactly and is not complicated by the difficulties one encounters in bound states of intersecting branes, such as the D1-D5-brane system [41].

$S O(6)$ invariance and power counting in the string coupling greatly restrict the possible couplings between the bulk and world-volume fields in the interaction part of the action $S_{\text {int }}$. In both the semi-classical and world-volume computations, the dimensionless expansion parameter is not $N g_{\text {str }}$ but rather $(\omega R)^{4}$. A cross-section which involves $n$ powers of this parameter arises from a coupling of the bulk field to a local operator $\mathcal{O}$ of dimension $n+4$.

The leading bosonic terms in the action for a single 3-brane in a type IIB supergravity background are [42,43]

$$
S_{3}=-T_{3} \int d^{4} x\left(\sqrt{-\operatorname{det}\left(\hat{g}+e^{-\phi / 2} \mathcal{F}\right)}+\frac{1}{4 !} \epsilon^{\alpha \beta \sigma \rho} \hat{C}_{\alpha \beta \sigma \rho}+\frac{1}{2} \hat{C}_{\alpha \beta} \tilde{\mathcal{F}}^{\alpha \beta}+\frac{1}{4} C \mathcal{F}_{\alpha \beta} \tilde{\mathcal{F}}^{\alpha \beta}\right),
$$

where

$$
\mathcal{F}_{\alpha \beta}=F_{\alpha \beta}+\hat{B}_{\alpha \beta}, \quad \tilde{F}^{\alpha \beta}=\frac{1}{2} \epsilon^{\alpha \beta \sigma \rho} F_{\sigma \rho}, \quad \hat{g}_{\alpha \beta}=g_{M N} \partial_{\alpha} X^{M} \partial_{\beta} X^{N} \text {, etc. },
$$


and the background fields are functions of $X^{M}$. In the static gauge $\left(X^{\alpha}=x^{\alpha}, \alpha=0, \ldots, 3\right)$ one has

$$
\hat{g}_{\alpha \beta}=g_{\alpha \beta}+2 g_{i(\alpha} \partial_{\beta)} X^{i}+g_{i j} \partial_{\alpha} X^{i} \partial_{\beta} X^{j}
$$

In flat space the fermionic terms may be found by replacing $\hat{g}_{\alpha \beta}+e^{-\phi / 2} \mathcal{F}_{\alpha \beta}$ by [39,40]

$$
\hat{g}_{\alpha \beta}+e^{-\phi / 2}\left[\mathcal{F}_{\alpha \beta}-2 \bar{\Psi}\left(\Gamma_{\alpha}+\Gamma_{i} \partial_{\alpha} X^{i}\right) \partial_{\beta} \Psi+\bar{\Psi} \Gamma^{i} \partial_{\alpha} \Psi \bar{\Psi} \Gamma_{i} \partial_{\beta} \Psi\right]
$$

where $\Psi$ is the $d=10$ Majorana-Weyl spinor (which can be split into four $d=4$ Majorana spinors $\psi_{I}$ in (3.4)) and $\Gamma_{M}$ are the $d=10$ Dirac matrices.

The leading-order interaction of the dilaton with world-volume fields implied by (3.5) was discussed in [23]. The coupling of the RR scalar $C$ is similar, being related by $S L(2, R)$ duality. The coupling of the gravitons polarized parallel to the brane, $h_{\alpha \beta}=g_{\alpha \beta}-\eta_{\alpha \beta}$, to the bosonic world-volume fields can be deduced by expanding the action (3.5). At the leading order it is given by $\frac{1}{2} h^{\alpha \beta} T_{\alpha \beta}^{\text {bosons }}$ where $T_{\alpha \beta}^{\text {bosons }}$ is the energy-momentum tensor of $A_{\alpha}$ and $X^{i}$. The only possible supersymmetric extension is for $h_{\alpha \beta}$ to couple to the complete energy-momentum tensor corresponding to (3.4).

Generalizing to $U(N)$, we find that the part of $S_{\text {int }}$ that is relevant to the leading-order absorption processes we wish to consider is 3

$$
S_{\mathrm{int}}=T_{3} \int d^{4} x\left[\operatorname{Tr}\left(\frac{1}{4} \phi F_{\alpha \beta}^{2}-\frac{1}{4} C F_{\alpha \beta} \tilde{F}^{\alpha \beta}\right)+\frac{1}{2} h_{\alpha \beta} T_{\alpha \beta}\right]
$$

where

$$
T_{\alpha \beta}=\operatorname{Tr}\left[F_{\alpha}^{\gamma} F_{\beta \gamma}-\frac{1}{4} \eta_{\alpha \beta} F_{\gamma \delta}^{2}-\frac{i}{2} \bar{\psi}^{I} \gamma_{(\alpha} \partial_{\beta)} \psi_{I}+\partial_{\alpha} X^{i} \partial_{\beta} X^{i}-\frac{1}{2} \eta_{\alpha \beta}\left(\partial_{\gamma} X^{i}\right)^{2}\right]
$$

Let us first consider an off-diagonal graviton polarized along the brane, say $h_{x y}$, which is an example of a traceless perturbation $h_{a b}$ whose quadratic action is given in (2.8)). From (3.6) one can read off the invariant amplitudes for absorption into two scalars, two fermions, or two gauge bosons:

$$
\begin{array}{ll}
\text { scalars: } & \mathcal{M}=-\sqrt{2 \kappa_{10}^{2}}\left(p_{1 x} p_{2 y}+p_{1 y} p_{2 x}\right) \\
\text { fermions: } & \mathcal{M}=-\frac{1}{2} \sqrt{2 \kappa_{10}^{2}} \bar{v}\left(-p_{1}\right)\left(\gamma_{x} p_{2 y}+\gamma_{y} p_{2 x}\right) u\left(p_{2}\right) \\
\text { gauge bosons: } & \mathcal{M}=-\sqrt{2 \kappa_{10}^{2}}\left(f_{x}^{(1) \beta} f_{y \beta}^{(2)}+f_{y}^{(1) \beta} f_{x \beta}^{(2)}\right)
\end{array}
$$

3 We ignore the fermionic couplings like $\phi \bar{\psi}^{I} \gamma^{\alpha} \partial_{\alpha} \psi_{I}$ and similar ones for $C$ and $h_{\alpha \beta}$ which are proportional to the fermionic equations of motion and thus give vanishing contribution to the S-matrix elements. 
where $p_{1}$ and $p_{2}$ are the momenta of the outgoing particles, and the field strength polarization tensors $f_{\alpha \beta}^{(s)}$ are given by

$$
f_{\alpha \beta}^{(s)}=i p_{s \alpha} \epsilon_{\beta}^{(s)}-i p_{s \beta} \epsilon_{\alpha}^{(s)}
$$

Summation over the spins of the outgoing particles can be performed using

$$
\begin{gathered}
\sum_{s} u_{(s)}(p) \bar{u}_{(s)}(p)=\not p, \quad \sum_{s} v_{(s)}(p) \bar{v}_{(s)}(p)=-\not p \\
\sum_{s} \epsilon_{\alpha}^{(s)} \epsilon_{\beta}^{(s) *}=\eta^{\alpha \beta} .
\end{gathered}
$$

Summing as well over different species of particles available (six different $X^{i}$, for example), one obtains

$$
\begin{array}{ll}
\text { scalars: } & |\overline{\mathcal{M}}|^{2}=3 \kappa_{10}^{2} \omega^{4} n_{x}^{2} n_{y}^{2} \\
\text { fermions: } & \overline{\mid \mathcal{M}}^{2}=\kappa_{10}^{2} \omega^{4}\left(n_{x}^{2}+n_{y}^{2}-4 n_{x}^{2} n_{y}^{2}\right) \\
\text { gauge bosons: } & |\overline{\mathcal{M} \mid}|^{2}=\kappa_{10}^{2} \omega^{4}\left(1-n_{x}^{2}-n_{y}^{2}+n_{x}^{2} n_{y}^{2}\right)
\end{array}
$$

where $\vec{n}$ is the direction of one of the outgoing particles. In (3.11) we have anticipated conservation of energy and momentum by setting $\vec{p}_{1}+\vec{p}_{2}=0$ and $\omega_{1}+\omega_{2}=\omega$. It is remarkable that the sum of these three quantities is independent of $\vec{n}$. Thus, if one performs the spin sums not just over all polarizations and species of particles of a given spin, but rather over all the states in the $\mathcal{N}=4$ super-multiplet, the result is isotropic:

$$
\overline{\mid \mathcal{M}}^{2}=\kappa_{10}^{2} \omega^{4}
$$

The absorption cross-section is evaluated from $\overline{\mid \mathcal{M}}^{2}$ in precisely the same way that decay rates of massive particles are calculated in conventional 4-dimensional field theories:

$$
\sigma_{3 \text { abs }}=\frac{N^{2}}{2} \frac{1}{2 \omega} \int \frac{d^{3} p_{1}}{(2 \pi)^{3} 2 \omega_{1}} \frac{d^{3} p_{2}}{(2 \pi)^{3} 2 \omega_{2}}(2 \pi)^{4} \delta^{4}\left(q-\sum_{i} p_{i}\right) \overline{\mid \mathcal{M}}^{2}
$$

The leading factor of $N^{2}$ accounts for the multiple branes; the $1 / 2$ is present because the outgoing particles are identical (this is true also of the fermions because we are working with Majorana spinors). The cross-section following from (3.12) agrees with the semiclassical $\ell=0$ result $(3.2)$ :

$$
\sigma_{3 \text { abs }}=\frac{\kappa_{10}^{2} \omega^{3} N^{2}}{32 \pi}=\sigma_{3 \text { class. }}
$$

where we have used the relation (3.3) between $N$ and $R$. 
Compared to the off-diagonal graviton, the calculation of the cross-section for the RR scalar is relatively simple. Inspection of the leading order amplitudes for absorption of a dilaton and a RR scalar makes it obvious that they have the same cross-section:

$$
\begin{aligned}
& \text { dilaton: } \quad \mathcal{M}=\frac{1}{2} \sqrt{2 \kappa_{10}^{2}} f_{\alpha \beta}^{(1)} f^{(2) \alpha \beta} \\
& \text { RR scalar: } \quad \mathcal{M}=-\frac{1}{2} \sqrt{2 \kappa_{10}^{2}} f_{\alpha \beta}^{(1)} \tilde{f}^{(2) \alpha \beta} .
\end{aligned}
$$

To show that $\overline{|\mathcal{M}|}^{2}=\kappa_{10}^{2} \omega^{2}$ in both cases, it suffices to prove the relation

$$
\sum_{\text {spins }} f_{\alpha \beta}^{(1)} f_{\gamma \delta}^{(1) *} f^{(2) \alpha \beta} f^{(2) \gamma \delta *}=\sum_{\text {spins }} f_{\alpha \beta}^{(1)} f^{(1) \gamma \delta *} \tilde{f}^{(2) \alpha \beta} \tilde{f}^{(2) \gamma \delta *}=8\left(p_{1} \cdot p_{2}\right)^{2} .
$$

The verification is straightforward algebra. The formula (3.13) applies as written to the $\mathrm{RR}$ scalar as well, so the agreement with the semi-classical calculation is clear.

\subsection{Higher partial waves}

A more difficult comparison is the absorption cross-section for higher partial waves. It is possible to argue, based on power counting and group theory, that the interaction term suggested in [23],

$$
S_{\mathrm{int}}^{\ell}=-T_{3} \int d^{4} x \frac{1}{4 \cdot \ell !} \partial_{i_{1}} \cdots \partial_{i_{\ell}} \phi \operatorname{Tr}\left(X^{i_{1}} \cdots X^{i_{\ell}} F_{\alpha \beta} F^{\alpha \beta}\right)
$$

is the only one that could possibly contribute at leading order to a given partial wave. It was shown in [23] that this term predicts a cross-section for the $\ell$-th partial wave whose scaling with $\omega$ and $N$ is in agreement with classical gravity. Here we show that, if we use the specific normalization given in (3.17), we obtain precise agreement for $\ell=0,1$ but disagreement for higher $\ell$. Later on we will argue that the disagreement for $\ell>1$ may be due simply to an incorrect normalization of the necessary effective action terms.

Let us restrict our attention to the dilaton. It will be obvious that all our arguments apply equally well to the RR scalar, and perhaps with a bit more attention to details to the off-diagonal gravitons. The absorption cross-section (3.2) is of order $\kappa_{10}^{\ell+2}$ for the $\ell$-th partial wave. Processes which can contribute to this absorption at leading order must be mediated by an operator $\mathcal{O}$ which involves $\ell+2$ fields. All interaction terms which involve the dilaton must include at least two powers of $F_{\alpha \beta}$ because of the restricted way in which the dilaton enters the DBI action. Since the gauge bosons are neutral under $S O(6)$, the other $\ell$ fields must be responsible for balancing the $S O(6)$ transformation properties of the $\ell$-th partial wave. We want to argue that the only way this can be done is to use $\ell$ powers of the scalar fields $X^{i}$. To do this it is necessary to know someting about the addition 
rules for representations of $S O(6)=S U(4)$. The $S U(4)$ Young tableaux for the fields in question are

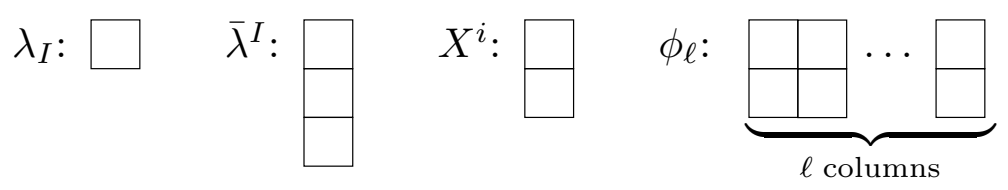

where $\phi_{\ell}$ stands for the $\ell$-th partial wave. There is a complicated general procedure known as the Littlewood-Richardson rule for taking tensor products of representations of $S U(N)$. It becomes much simpler when one of the factors is a fundamental representation ( $k$ boxes in a single column) 44]. In that case, one adds these $k$ boxes to the other factor's Young tableaux in all possible ways, modulo the restriction that not more than one box can be added to any given row. Then one eliminates all columns containing $N$ boxes. Using this rule it is easy to show that with $\ell$ fundamental representations as the factors in a tensor product, it is impossible to obtain the tableau for $\phi_{\ell}$ unless all the factors are 6 representations.

A shorter argument can be made based on purely dimensional grounds. The dimension of $\mathcal{O}$ must be $\ell+4$ or the cross-section would be suppressed by extra powers of $\omega$. Since $F_{\alpha \beta}^{2}$ has dimension 4 , the other $\ell$ fields must add $\ell$ to the total dimension of $\mathcal{O}$. The only possibility is $\ell$ scalars.

The upshot is that the only possible term in the action which can contribute at leading order to the absorption of a dilaton in the $\ell$-th partial wave is, up to normalization, given by (3.17). The normalization given there is the one arising from Taylor expanding $\phi(X)$. Actually, this action is not quite the one we want for $\ell>1$ : in addition to mediating the process which contributes at leading order to the absorption of the $\ell$-th partial wave, it makes subleading contributions to lower partial waves. In order to isolate the $\ell$-th partial wave, the product $X^{i_{1}} \cdots X^{i_{\ell}}$ should be replaced by an expression which transforms irreducibly under $S O(6)$. For $\ell=2$ and $\ell=3$, the appropriate replacements are

$$
\begin{aligned}
& \ell=2: \quad X^{i_{1}} X^{i_{2}} \rightarrow X^{i_{1}} X^{i_{2}}-\frac{1}{6} \delta^{i_{1} i_{2}} X^{2} \\
& \ell=3: \quad X^{i_{1}} X^{i_{2}} X^{i_{3}} \rightarrow X^{i_{1}} X^{i_{2}} X^{i_{3}}-\frac{3}{8} \delta^{\left(i_{1} i_{2}\right.} X^{\left.i_{3}\right)} X^{2} .
\end{aligned}
$$

One thus subtracts an $\ell=0$ contribution from the putative $\ell=2$ term in (3.17), and an $\ell=1$ contribution from $\ell=3$. The whole Taylor series can be thus reshuffled.

Because the replacements in (3.19) project out a final state with definite $\ell$, it is permissible to let the initial dilaton wave-function be $e^{-i \omega\left(t-x^{1}\right)}$ as usual. The derivatives

4 As we argue below, it is possible that this local expansion is not consistent with the string amplitude calculations. This may remove the discrepancy for $\ell>1$. 
$\partial_{i}$ in 3.17$)$ can then be replaced by $i \omega \delta_{i}^{1}$. The spin-summed amplitudes squared for absorption of the first few partial waves are

$$
\begin{aligned}
& \ell=0: \quad \overline{\mid \mathcal{M}}^{2}=4 \kappa_{10}^{2}\left(p_{1} \cdot p_{2}\right)^{2} \\
& \ell=1: \quad \overline{\mid \mathcal{M}}^{2}=\frac{4}{\sqrt{\pi}} \kappa_{10}^{3} \omega^{2}\left(p_{1} \cdot p_{2}\right)^{2} \\
& \ell=2: \quad \overline{\mid \mathcal{M}}^{2}=\frac{10}{3 \pi} \kappa_{10}^{4} \omega^{4}\left(p_{1} \cdot p_{2}\right)^{2} \\
& \ell=3: \quad \overline{\mid \mathcal{M}}^{2}=\frac{5}{2 \pi^{3 / 2}} \kappa_{10}^{5} \omega^{6}\left(p_{1} \cdot p_{2}\right)^{2} .
\end{aligned}
$$

As before, $p_{1}$ and $p_{2}$ are the momenta of the outgoing gauge bosons. The gauge bosons are identical particles, as are the $\ell$ outgoing scalars, whose momenta will be labeled $p_{3}, \ldots$, $p_{\ell+2}$. To avoid over-counting in the integral over phase space, we must include a factor of $1 /(2 \cdot \ell !)$ in the expression for the cross-section. The cross-section also includes an explicit factor of $1 /(2 \omega)$ from the normalization of the incoming dilaton. Altogether,

$$
\sigma_{\mathrm{D}}^{\ell}=\frac{N^{\ell+2}}{2 \cdot \ell !} \frac{1}{2 \omega} \int \frac{d^{3} p_{1}}{(2 \pi)^{3} 2 \omega_{1}} \cdots \frac{d^{3} p_{\ell+2}}{(2 \pi)^{3} 2 \omega_{\ell+2}}(2 \pi)^{4} \delta^{4}\left(q-\sum_{i} p_{i}\right) \overline{\mid \mathcal{M}}^{2} .
$$

The momentum integrations take the following form:

$$
I_{\ell}=\int \frac{d^{3} p_{1}}{2 \omega_{1}} \cdots \frac{d^{3} p_{\ell+2}}{2 \omega_{\ell+2}} \delta^{4}\left(q-\sum_{i} p_{i}\right)\left(p_{1} \cdot p_{2}\right)^{2}=\frac{3 \pi^{\ell+1}}{2^{\ell+1}} \frac{\omega^{2 \ell+4}}{(\ell+2) !(\ell+3) !} .
$$

The quickest way to establish (3.22) is to Fourier transform to position space.

The final results for the first few $\ell$ are

$$
\begin{aligned}
\sigma_{\mathrm{D}-\text { brane }}^{\ell=0} & =\sigma_{\text {class. }}^{\ell=0} \\
\sigma_{\mathrm{D}-\text { brane }}^{\ell=1} & =\sigma_{\text {class. }}^{\ell=1} \\
\sigma_{\mathrm{D}-\text { brane }}^{\ell=2} & =\frac{9}{5} \sigma_{\text {class. }}^{\ell=2} \\
\sigma_{\mathrm{D}-\text { brane }}^{\ell=3} & =\frac{24}{5} \sigma_{\text {class. }}^{\ell=3} .
\end{aligned}
$$

If the $\ell=2$ and $\ell=3 \mathrm{D}$-brane cross-sections had been smaller than the corresponding classical results, one might have wondered if the argument around (3.18) might possibly be invalidated by some peculiar interaction. But any such additional interaction could only increase the D-brane cross-section, making the disagreement even worse.

It is worth noting that nowhere in the literature have higher partial waves been compared with complete success between D-brane models and General Relativity. Agreement up to numerical factors was obtained in [45] for effective string models of four- and fivedimensional black holes, and independently in [38] for the five-dimensional case; but in the 
absence of a well articulated prescription for coupling the effective string to the bulk fields it is difficult to tell much about numerical coefficients.

The agreement of the $\ell=1$ cross-section certainly encourages us to believe that the D-brane description is capable of handling partial waves correctly. On the level of effective field theory this may seem peculiar because, in this low-energy description, the D-brane has no thickness. How can an object with no extent in transverse dimensions absorb particles with angular momentum? Power counting and group theory alone seem to dictate the answer (3.17), up to normalization.

Fortunately, with threebranes the string theory prescription for the couplings to external fields is more directly accessible than for other solitonic models of black holes. While it does indeed appear that $\ell>1$ partial waves present a test which the DBI action, supplemented by the prescription of [23] to obtain the normalization of (3.17) via a Taylor expansion, fails to pass, we expect that a proper string theoretic treatment will once again yield agreement. For $\ell=1$, a full-fledged disk amplitude computation verifies both the form of (3.17) and the normalization shown. The $\ell=1$ disk amplitude, which involves one bulk insertion and three boundary operators, is easy to deal with because the insertion of one scalar vertex operator on the boundary simply generates a space-time translation. When more than one such operators are present, one encounters singularities in their mutual collisions that need a careful treatment. The necessary calculations look quite complicated: for example, computing the full $\ell=2$ amplitude would be equivalent via the prescription of [16 to computing a six point type I disk amplitude. They nevertheless seem highly worthwhile as a means to refine our understanding of the world-volume action.

\subsection{Absorption of Higher Spin Particles}

In the previous subsections we dealt exclusively with scalar particles. Of course, in string theory one also encounters particles of higher spin, such as the gravitons. The necessary string theory calculations for them are of the same order of complexity as for scalars. The comparison with classical gravity is complicated by the fact that it is difficult in general to decouple the necessary equations. This problem has been studied to some extent for $d=4$ black holes (see [46] and references therein). Nothing is known, however, about the $d=7$ black holes relevant to this discussion. While we hope to come back to the classical aspect of this problem in the future, below we give a brief discussion of the effective action results.

In 23] absorption of gravitons polarized transversely to the threebrane was studied with the result that the cross-section has the same value (3.14) as the scalar cross-section. This is suggestive of a residual supersymmetry relation between particles of different spin: while the supersymmetry is broken by the incident energy, it may still constrain the lowenergy limit of the cross-section. In this section we consider particles that are vectors from 
the $d=7$ point of view and find that their absorption cross-section is $2 / 3$ times that of the minimally coupled scalar.

One example of a state which gives rise to a vector particle upon dimensional reduction to $d=7$ is obtained from the $B_{M N}$ (see (A.4)). The spatial and time components of the vector potential are given by $\left(B_{\alpha i}, B_{\alpha 0}\right)$, where $\alpha$ is one of the longitudinal directions (say, $\alpha=x)$. We will choose the gauge $B_{\alpha 0}=0$.

In the world-volume action, the leading term responsible for absorption is

$$
-T_{3} \int d^{4} x \frac{1}{4}\left(F_{\alpha \beta}+\hat{B}_{\alpha \beta}\right)^{2}
$$

For the mixed polarization that we are interested in,

$$
\hat{B}_{\alpha \beta}=B_{\alpha i} \partial_{\beta} X^{i}-B_{\beta i} \partial_{\alpha} X^{i}
$$

Upon rescaling the world-volume fields by $\sqrt{T}_{3}$ and generalizing to $U(N)$, the coupling is given by

$$
-\int d^{4} x B_{\alpha i} \operatorname{Tr}\left(\partial_{\beta} X^{i} F^{\alpha \beta}\right)
$$

Choosing one particular component, e.g., $\alpha=x, i=7$, and using the properly normalized field, $B_{x 7} /\left(\sqrt{2} \kappa_{10}\right)$ (cf. (A.4)), we get the amplitude

$$
\mathcal{M}=\sqrt{2} \kappa_{10} p_{1}^{\beta}\left(p_{2 x} \epsilon_{\beta}-p_{2 \beta} \epsilon_{1}\right)
$$

Summing over polarizations, we find that

$$
\overline{|\mathcal{M}|}^{2}=2 \kappa_{10}^{2} \frac{\omega^{4}}{4}\left(1-n_{x}^{2}\right)
$$

Is there an additional contribution of the same order due to the world-volume fermions? If we use the prescription of [39,40 to supersymmetrize the action (3.5), then we find that the answer is negative: the leading coupling to the Majorana-Weyl fermion $\Psi$ is via a dimension 6 operator,

$$
\sim B_{\alpha j} \partial_{\beta} X^{j} \bar{\Psi} \Gamma_{[\alpha} \partial_{\beta]} \Psi
$$

Thus, it appears that the direction dependence of the absorption process does not cancel out for the $d=7$ vectors. Since $\left\langle n_{x}^{2}\right\rangle=1 / 3$, we find, multiplying (3.25) by the number of final states and the phase space factor, $1 /(16 \pi \omega)$, that the total vector cross-section is given by $2 / 3$ times the cross-section of a minimally coupled scalar, (3.14).

It would be interesting to confirm the absence of a leading fermionic contribution using the string amplitude calculations of [17]. Perhaps the mixing of perturbations around the 3 -brane, discussed in the Appendix, can explain the factor of $2 / 3$. 


\section{Comparing absorption cross-sections for M-branes}

A remarkable aspect of the agreement between the string theoretic and the classical results for threebranes is that it holds exactly for any value of $N$, including $N=1$. As explained in the introduction, the classical geometry should be trusted only in the limit $g_{\text {str }} N \rightarrow \infty$. Thus, the agreement of the absorption cross-sections for $N=1$ suggests that our calculations are valid even in the limit $g_{\mathrm{str}} \rightarrow \infty$, provided that $g_{\mathrm{str}} \alpha^{\prime 2} \omega^{4}$ is kept small. The supersymmetric non-renormalization theorems are probably at work here, insuring that there are no string loop corrections.

In this section we would like to ask whether the exact agreement between the absorption cross-sections is also found for the twobranes and fivebranes of M-theory. These branes have much in common with the self-dual threebrane of type IIB theory: their entropies scale with the temperature in agreement with the scaling for a gas of massless fields on the world-volume [19], while the scaling of their absorption cross-sections with $\omega$ agrees with estimates from the world-volume effective theory [23]. While the effective actions for one twobrane [47] and one fivebrane 48] are now known in some detail, their generalizations to $N>1$ remain somewhat obscure.5 In this section we compare the cross-sections for $N=1$ and find that, in contrast to the threebranes, there is no exact agreement in the normalizations. This is probably due to the fact that M-theory has no parameter like $g_{\mathrm{str}}$ that can be dialed to make the classical solution reliable.

First we discuss absorption of longitudinally polarized gravitons by an twobrane. The massless fields in the effective action are 8 scalars and 8 Majorana fermions. The longitudinal graviton couples to the energy momentum tensor on the world-volume, $T_{\alpha \beta}$. The terms in the effective action necessary to describe the absorption of $h_{x y}$ are $(i=3, \ldots, 10$; $I=1, \ldots, 8)$

$$
\begin{aligned}
& S_{2}=T_{2} \int d^{3} x\left[-\frac{1}{2} \partial_{\alpha} X^{i} \partial^{\alpha} X^{i}+\frac{i}{2} \bar{\psi}^{I} \gamma^{\alpha} \partial_{\alpha} \psi^{I}\right. \\
& \left.+\sqrt{2} \kappa_{11} h_{x y}\left(\partial_{x} X^{i} \partial_{y} X^{i}-\frac{i}{4} \bar{\psi}^{I}\left(\gamma_{x} \partial_{y}+\gamma_{y} \partial_{x}\right) \psi^{I}\right)\right]
\end{aligned}
$$

where $h_{x y}$ is the canonically normalized field which enters the $D=11$ space-time action as (cf. (2.12))

$$
-\frac{1}{2} \int d^{11} x \partial_{M} h_{x y} \partial^{M} h_{x y}
$$

The absorption cross-section is found using the Feynman rules in a way analogous to the threebrane calculation of section 3 . For the 8 scalars, we find that the matrix element

5 It is believed, for instance, that $N>1$ coincident fivebranes are described by non-trivial conformally invariant theories in $5+1$ dimensions. Comparisons with classical gravity of the kind made in [19,23] and here are among the ways of learning more about this theory. 
squared (with all the relevant factors included) is

$$
\frac{\kappa_{11}^{2} \omega^{4}}{2} 4 n_{x}^{2} n_{y}^{2},
$$

where $\vec{n}$ is the unit vector in the direction of one of the outgoing particles. For the 8 Majorana fermions, the corresponding object summed over the final polarizations is

$$
\frac{\kappa_{11}^{2} \omega^{4}}{2}\left(n_{x}^{2}-n_{y}^{2}\right)^{2}
$$

Adding them up, we find that the dependence on direction cancels out, just as in the threebrane case. The sum must be multiplied by the phase space factor

$$
\frac{1}{2 \omega} \frac{1}{2 \pi} \int \frac{d^{2} p_{1}}{2 \omega_{1}} \int \frac{d^{2} p_{2}}{2 \omega_{2}} \delta^{2}\left(\vec{p}_{1}+\vec{p}_{2}\right) \delta\left(\omega_{1}+\omega_{2}-\omega\right)=\frac{1}{8 \omega^{2}},
$$

so that the total cross-section is

$$
\sigma_{2 \text { abs }}=\frac{\kappa_{11}^{2} \omega^{2}}{16} .
$$

This does not agree with the classical result for $N$ set to 1 ,

$$
\sigma_{2 \text { class. }}=\frac{2 \pi^{4}}{3} \omega^{2} R^{9}=\frac{2 \sqrt{2}}{3 \pi} \kappa_{11}^{2} \omega^{2} N^{3 / 2},
$$

where we have used the twobrane charge quantization to express $R^{9}$ in terms of $N$. Notice that even the power of $\pi$ does not match. This situation is reminiscent of the discrepancy in the near-extremal entropy where the relative factor was a transcendental number involving $\zeta(3)$ [19].

Now we show that, just as for the threebrane, the transversely polarized gravitons have the same absorption cross-section as the longitudinally polarized gravitons. The coupling of $h_{67}$ to scalars is given by

$$
-T_{2} \int d^{3} x \sqrt{2} \kappa_{11} h_{67} \partial_{\alpha} X^{6} \partial^{\alpha} X^{7},
$$

while pairs of fermions are not produced because the coupling to them vanishes on shell. The matrix element squared is $\kappa_{11}^{2} \omega^{4} / 2$. Multiplying this by the phase space factor, we again find the cross-section (4.2).

Now we turn to the fivebrane. The massless fields on the fivebrane form a tensor multiplet consisting of 5 scalars, 2 Weyl fermions and the antisymmetric tensor $\mathcal{B}_{\alpha \beta}$ with anti-selfdual strength [49]. The transverse gravitons are again the easier case because they produce pairs of scalars only (the coupling to 2 fermions vanishes on shell). The necessary 
coupling of $h_{67}$ is similar to (4.3), $-T_{5} \int d^{6} x \sqrt{2} \kappa_{11} h_{67} \partial_{\alpha} X^{6} \partial^{\alpha} X^{7}$. The matrix element squared is $\kappa_{11}^{2} \omega^{4} / 2$, while the phase space factor is now

$$
\frac{1}{2 \omega} \frac{1}{(2 \pi)^{4}} \int \frac{d^{5} p_{1}}{2 \omega_{1}} \int \frac{d^{5} p_{2}}{2 \omega_{2}} \delta^{5}\left(\vec{p}_{1}+\vec{p}_{2}\right) \delta\left(\omega_{1}+\omega_{2}-\omega\right)=\frac{\omega}{2^{7} \cdot 3 \pi^{2}},
$$

so that the absorption cross-section is

$$
\sigma_{5 \text { class. }}=\frac{\kappa_{11}^{2} \omega^{5}}{2^{8} \cdot 3 \pi^{2}}
$$

To discuss the absorption of longitudinally polarized gravitons, $h_{x y}$, we need the action $(i=6, \ldots, 10 ; \quad I=1,2)$

$$
\begin{aligned}
& S=T_{5} \int d^{6} x\left[-\frac{1}{2} \partial_{\alpha} X^{i} \partial^{\alpha} X^{i}-\frac{1}{12} \mathcal{H}_{\alpha \beta \gamma}^{2}+i \bar{\psi}^{I} \gamma^{\alpha} \partial_{\alpha} \psi^{I}\right. \\
& \left.+\sqrt{2} \kappa_{11} h_{x y}\left(\partial_{x} X^{i} \partial_{y} X^{i}+\frac{1}{2} \mathcal{H}_{x \beta \gamma}^{-} \mathcal{H}_{y}^{-\beta \gamma}-\frac{i}{2} \bar{\psi}^{I}\left(\gamma_{x} \partial_{y}+\gamma_{y} \partial_{x}\right) \psi^{I}\right)\right]
\end{aligned}
$$

To describe interaction of the anti-selfdual antisymmetric tensor with external field we follow the covariant approach of [50] using the standard 'unconstrained' propagator for $\mathcal{B}_{\alpha \beta}$ and replacing $\mathcal{H}$ by its anti-selfdual part $\mathcal{H}^{-}=\frac{1}{2}(\mathcal{H}-\tilde{\mathcal{H}})$ in the vertices.

For the 5 scalars, we find that the matrix element squared (with all the relevant factors included) is

$$
\frac{\kappa_{11}^{2} \omega^{4}}{2} \frac{5}{2} n_{x}^{2} n_{y}^{2}
$$

For the 2 Weyl fermions, the corresponding object summed over the final polarizations is

$$
\frac{\kappa_{11}^{2} \omega^{4}}{2}\left(n_{x}^{2}+n_{y}^{2}-4 n_{x}^{2} n_{y}^{2}\right)
$$

Finally, the contribution of the anti-selfdual gauge field turns out to be equal to that of the usual, unconstrained $\mathcal{H}_{\alpha \beta \gamma}$ divided by 2. The matrix element squared is, therefore,

$$
|\mathcal{M}|^{2}=\frac{1}{2} 2 \kappa_{11}^{2} \frac{1}{4} \sum\left[\mathcal{H}_{x \beta \gamma}^{(1)} \mathcal{H}_{y}^{(2) \beta \gamma}+\mathcal{H}_{x \beta \gamma}^{(2)} \mathcal{H}_{y}^{(1) \beta \gamma}\right] \mathcal{H}_{x \alpha \delta}^{(1) *} \mathcal{H}_{y}^{(2) \alpha \delta *}
$$

6 It is interesting to observe that, if we consider an $\mathcal{N}=1$ multiplet consisting of 1 Weyl fermion and 4 scalars, then the 'tensor term' $n_{x}^{2} n_{y}^{2}$ cancels out in the direction dependence. The same cancellation occurs for the threebrane (both for the $\mathcal{N}=1$ vector multiplet and for the $\mathcal{N}=1$ hypermultiplet).

7 Since the propagator of $\mathcal{B}_{\alpha \beta}$ is taken to be non-chiral, it is sufficient to do the replacement $\mathcal{H} \mathcal{H} \rightarrow \mathcal{H}^{-} \mathcal{H}^{-}$in only one of the two stress tensor factors in $|\mathcal{M}|^{2}$. The relevant part of $\mathcal{H}^{-} \mathcal{H}^{-}$ is $\frac{1}{4}(\mathcal{H} \mathcal{H}+\tilde{\mathcal{H}} \tilde{\mathcal{H}})$ which is equal to $\frac{1}{2} \mathcal{H} \mathcal{H}$ for off-diagonal components of the stress tensor. 
where we have also included $1 / 2$ because the outgoing particles are identical. Sums over polarizations are to be performed with

$$
\sum \epsilon_{\alpha \beta} \epsilon_{\gamma \delta}^{*}=\eta_{\alpha \gamma} \eta_{\beta \delta}-\eta_{\alpha \delta} \eta_{\beta \gamma}
$$

The entire calculation is lengthy, but the end result is simple, 8

$$
\overline{\mid \mathcal{M}}^{2}=\frac{\kappa_{11}^{2} \omega^{4}}{2}\left(1-n_{x}^{2}-n_{y}^{2}+\frac{3}{2} n_{x}^{2} n_{y}^{2}\right)
$$

Adding up the contributions of the entire tensor multiplet, we find that all the direction-dependent terms cancel out, just as they did for the threebrane and the twobrane. Multiplying by the phase space factor (4.4), we find that the total cross-section for the longitudinally polarized gravitons is again given by (4.5). This turns out to be a factor of 4 smaller than the classical result,

$$
\sigma_{5 \text { class. }}=\frac{2 \pi^{3}}{3} \omega^{5} R^{9}=\frac{\kappa_{11}^{2} N^{3} \omega^{5}}{2^{6} \cdot 3 \pi^{2}}
$$

evaluated for $N=1$, i.e. $\sigma_{5}$ abs $=\frac{1}{4} \sigma_{5}^{(N=1)}$ class. . This discrepancy is relatively minor and is of a kind that could easily be produced by a calculational error. However, having checked our calculations a number of times, we believe that the factor of 4 discrepancy is real.

A conclusion that we may draw from this section is that, although the single M-brane cross-sections scale with the energy in the same way as the classical cross-sections, the normalizations do not agree. The fivebrane comes much closer to agreement than the twobrane, which may be connected to the fact that the fivebrane supergravity solution is completely non-singular. For $N=1$, however, the curvature of the solution is of order of the 11-dimensional Planck scale. Obviously, the 11-dimensional supergravity is at best a lowenergy approximation to M-theory. The M-theory effective action should contain higherderivative terms weighted by powers of $\kappa_{11}$, by analogy with the $\alpha^{\prime}$ and $g_{\text {str }}$ expansions of the string effective action. Thus, for $N=1$, the classical solution may undergo corrections of order one which we believe to be the source of the discrepancy. For large $N$, however, we expect the M-theory cross-section to agree exactly with the classical cross-section. We hope that these considerations will serve as a useful guide in constructing the world volume theory of $N$ coincident fivebranes.

8 This answer passes also a number of heuristic checks. For instance, for the $\mathcal{N}=1$ multiplet including the gauge field, 1 scalar and 1 Weyl fermion, the $n_{x}^{2} n_{y}^{2}$ terms again cancels out. 


\section{Conclusions}

In this paper we have provided new evidence, furthering the earlier results of [23], that there exists exact agreement between the classical and the D-brane descriptions of the self-dual threebrane of type IIB theory. The specific comparisons that we have carried out involve probing an extremal threebrane with low-energy massless quanta incident from the outside. As argued in [23] and here, the great advantage of the threebrane is that both the perturbative string theory and the classical supergravity calculations are under control and yield expansions in the same dimensionless expansion parameter $(\omega R)^{4} \sim N g_{\mathrm{str}} \alpha^{\prime 2} \omega^{4}$, which may be kept small.

While the low-energy physics of the threebrane is described by a $\mathcal{N}=4 \mathrm{SYM}$ theory, probing it from the extra dimensions provides a new point of view and allows for a variety of interesting calculations. One example is the absorption of a graviton polarized parallel to the brane which, as discussed in section 3, couples to the energy-momentum tensor in $3+1$ dimensions. Because of the existence of the transverse momentum, the kinematics for this process is that of a decay of a massive spin-two particle into a pair of massless worldvolume modes. Summing over all the states in the $\mathcal{N}=4$ multiplet we find that the rate is completely isotropic, which is undoubtedly related to the conformal invariance of the theory. We should emphasize, however, that we are exploring the properties of this theory away from the BPS limit; therefore, they are not determined by supersymmetry alone. For this reason, we find it remarkable that the net absorption cross-section has the same value as that of the dilaton and the RR scalar, and which agrees with the cross-section found in classical supergravity.

We have also carried out similar explorations of the physics of M-branes. These studies are hampered, to a large extent, by insufficient understanding of the multiple coincident branes of M-theory. For a single twobrane, a formal application of classical gravity gives an answer which is off by a factor of $32 \sqrt{2} /(3 \pi)$, while for a single fivebrane - off only by a factor of 4.9 For the fivebrane the discrepancy is relatively minor which, we are tempted to speculate, is due to the fact that its classical geometry is completely non-singular, just like that of the threebrane. We should keep in mind, however, that for singly charged branes the quantum effects of M-theory are expected to be important, and the classical reasoning should not be trusted.

While in this paper we have probed 3-branes with massless particles, we may contemplate another interesting use of the 3-brane: it may be used as a probe by itself [51]. For example, $N$ coincident 3 -branes may be probed by a 3 -brane parallel to them. This situation is described by a $3+1$ dimensional $\mathcal{N}=4$ supersymmetric $U(N+1)$ gauge theory, with gauge symmetry broken to $U(N) \times U(1)$. A more complicated theory will arise if a

\footnotetext{
9 Curiously, both mismatch factors are of the same order, as $32 \sqrt{2} /(3 \pi) \approx 4.8$.
} 
3-brane is used to probe a stringy $d=4$ black hole. As was shown in [52,53, the $d=4$ extremal black holes with regular horizons (which are parametrised by 4 charges [54]) can be represented by $1 / 8$ supersymmetric configurations of four intersecting 3 -branes wrapped over a 6-torus. Following [55] one can find an action for a classical 3-brane probe moving in this geometry. If the probe is oriented parallel to one of the four source 3-branes, the resulting moduli space metric is

$$
d s_{6}^{2}=H_{1} H_{2} H_{3}\left(d r^{2}+r^{2} d \Omega_{2}^{2}\right)+H_{1} d y_{1}^{2}+H_{2} d y_{2}^{2}+H_{3} d y_{3}^{2}, \quad H_{i}=1+\frac{R_{i}}{r},
$$

where $y_{i}$ are toroidal coordinates transverse to the probe. In contrast to the case of the '5-brane+string + momentum' configuration describing $d=5$ regular extremal black holes with 3 charges [56] where one finds [57 that the non-compact part of the moduli space metric is multiplied by the product of two harmonic functions, here we obtain the product of three harmonic functions. This is, however, exactly what is needed to get the same nearhorizon $(r \rightarrow 0)$ behaviour as found in [57], i.e. that the 3-dimensional non-compact part of the moduli space metric becomes flat: $d s_{3}^{2} \rightarrow R_{1} R_{2} R_{3}\left(d \rho^{2}+\rho^{2} d \Omega_{2}^{2}\right), \quad \rho \equiv r^{-1 / 2} \rightarrow \infty$. This close similarity between a $d=5$ black hole probed by a string and a $d=4$ black hole probed by a 3-brane strongly suggests that one can obtain important information about these black holes by studying the corresponding 3-brane world-volume theory. This theory in the presence of intersecting branes is necessarily more intricate than the simpler, parallel brane case discussed in the main part of this paper.

Clearly, there is much work to be done before we claim a complete understanding of the remarkable duality between the D-brane and the classical descriptions of the threebrane. The complexities that affect the higher-spin classical equations remain to be disentangled. The numerical discrepancies for $\ell>1$ partial waves suggest an incomplete understanding of the low-energy effective action. As we have argued in section 3, a direct string calculation is necessary to check normalizations. And finally, we need to push our methods away from extremality where the physics is necessarily more complicated, involving thermal field theory in $3+1$ dimensions. We hope that detailed insight into such a theory will help explain the specific factors appearing in the near-extremal entropy [18.

We feel that further efforts in the directions mentioned above are worthwhile because

they offer a promise of building a theory of certain black holes in terms of a manifestly unitary theory - perturbative string theory.

\section{Acknowledgements}

We are grateful to V. Balasubramanian, C.G. Callan, and A. Hashimoto for useful discussions. This work was supported in part by DOE grant DE-FG02-91ER40671, the NSF Presidential Young Investigator Award PHY-9157482, and the James S. McDonnell Foundation grant No. 91-48. A.A.T. also acknowledges the support of PPARC and the European Commission TMR programme ERBFMRX-CT96-0045. 


\section{Appendix A. Comments on mixed perturbations}

In this Appendix we discuss the problem of mixing between different perturbations around the self-dual threebrane. We may attempt to classify various perturbations according to the power of the harmonic function $H(r)$ which appears in front of their kinetic term. For example, the off-diagonal graviton perturbations (the $d=7$ Kaluza-Klein vectors $\left.A_{\mu}^{a}=g^{a b} h_{b \mu}\right)$ have the following kinetic term

$$
\begin{aligned}
& S_{\text {vect.grav. }}=\frac{1}{2 \kappa_{10}^{2}} \int d^{10} x \sqrt{-g_{10}}\left(-\frac{1}{4} g_{a b} F^{a \mu \nu} F_{\mu \nu}^{b}+\ldots\right) \\
& =-\frac{1}{8 \kappa_{10}^{2}} \int d^{10} x H^{-1}(r)\left[F_{i j}^{a} F_{i j}^{a}-2 H(r) F_{0 j}^{a} F_{0 j}^{a}\right]+\ldots .
\end{aligned}
$$

Because of the extra factor of $H^{-1}$ in the kinetic term one expects that their low-energy absorption rate is suppressed compared to that for the minimally coupled massless scalars in (2.5), (2.8) (cf. [10]). However, Eq. (A.1) is not the complete action for the vector perturbations $\delta A_{\mu}^{a}$, as they 'mix' with fluctuations of $C_{4}$. The components of $C_{4}$ which vanish in the 3-brane background are $C_{\mu \nu a b}$ (antisymmetric 2-tensors in $d=7$ ) and $C_{\mu \nu \lambda a}$ (antisymmetric 3 -tensors dual to vectors in $d=7$ ). These two types of components are related by the self-duality condition,

$$
F_{i j k a b} \sim \epsilon_{a b c} \epsilon_{i j k l m n} F_{0 l m n c}, \quad F_{0 j k a b} \sim H^{-1} \epsilon_{a b c} \epsilon_{j k l m n i} F_{l m n i c} .
$$

The action for the quadratic fluctuations of the first field has the same structure as the scalar actions (2.5), (2.8) $\left(F_{\mu \nu \lambda a b}=3 \partial_{[\mu} C_{\nu \lambda] b c}\right)^{10}$

$$
\begin{gathered}
S_{\text {tens. }}=\frac{1}{2 \kappa_{10}^{2}} \int d^{10} x \sqrt{-g_{11}}\left(-\frac{1}{48} F_{\mu \nu \lambda a b} F^{\mu \nu \lambda a b}\right) \\
=-\frac{1}{96 \kappa_{10}^{2}} \int d^{10} x\left[F_{i j k a b} F_{i j k a b}-3 H(r) F_{0 j k a b} F_{0 j k a b}\right] .
\end{gathered}
$$

The coupling between $C_{\mu \nu a b}$ and the graviton perturbation $h_{\mu a}=\delta A_{\mu}^{a}$ originates from the $F_{5}^{2}$ term in (2.2) (or from the self-duality condition implying $F_{0 i j a b} \sim \epsilon_{a b c} \partial_{i} H^{-1} h_{j c}$ ) and has the following structure,

$$
\sim \epsilon_{a b c} H^{-1} \partial_{i} H F_{0 i j a b} h_{j c}
$$

10 The action for $C_{\mu \nu \lambda a}$ has an extra factor of $H^{-1}$ in front of $\left[F_{i j k l a} F_{i j k l a}-4 H(r) F_{0 j k l a} F_{0 j k l a}\right]$, and its absorption should be suppressed at low energies. This will follow also from world-volume considerations discussed below: while $C_{i j a b}$ couples to the 'marginal' $\left(d X^{i}\right)^{2}$ operator, $C_{i j k a}$ couples to the $\left(d X^{i}\right)^{3}$-term which produces a smaller cross-section at low energies. 
A similar pattern is found for the structure of the 2-nd rank antisymmetric tensor perturbations in (2.2). The fields $B_{a b}, C_{a b}$ (which become scalars in $d=7$ ) and $B_{\mu a}, C_{\mu a}$ (which become vectors in $d=7$ ) are non-trivially coupled pairwise ( $B_{a b}$ with $C_{\mu a}$ and $C_{a b}$ with $B_{\mu a}$ ) via the WZ-term in (2.1) and the 'cross-term' in $F_{5}^{2}$. Both contributions to the coupling have the same form because of the self-duality of the background value of $\partial C_{4}$. The kinetic part of their action is (cf. (2.5), (A.1), (A.2))

$$
\begin{aligned}
S_{\text {vect. }}= & \frac{1}{2 \kappa_{10}^{2}} \int d^{10} x\left(-\frac{1}{4} H(r)\left[\left(\partial_{i} B_{a b}\right)^{2}-H(r)\left(\partial_{0} B_{a b}\right)^{2}+\left(\partial_{i} C_{a b}\right)^{2}-H(r)\left(\partial_{0} C_{a b}\right)^{2}\right]\right. \\
& \left.-\left[\left(\partial_{[i} B_{j] a}\right)^{2}-2 H(r)\left(\partial_{[0} B_{i] a}\right)^{2}+\left(\partial_{[i} C_{j] a}\right)^{2}-2 H(r)\left(\partial_{[0} C_{i] a}\right)^{2}\right]\right), \quad(\text { A. } 4)
\end{aligned}
$$

and the mixing terms are

$$
\sim \epsilon_{a b c} \partial_{i} H\left(C_{a b} \partial_{[0} B_{i] c}-B_{a b} \partial_{[0} C_{i] c}\right) .
$$

While the scalars $B_{a b}, C_{a b}$ have extra factors of $H$ in front of their kinetic terms, the vectors $B_{\mu a}, C_{\mu a}$ have the standard kinetic terms as in (2.5). We hope that the mixing which affects these $d=7$ vectors is capable of reproducing the peculiar factor of $2 / 3$ multiplying their absorption cross-sections, found in section 3.4 using the effective field theory on world-volume.

Analogous observations can be made on the problem of mixing between different perturbations around the M-brane solutions. The Kaluza-Klein vector field has the action of the same form as (A.1) in the type IIB case, i.e. with the factor $H^{-1}$ in front of the kinetic term. In the twobrane (fivebrane) case it mixes (cf. (A.3)) with the electric (magnetic) component of the field strength of $C_{\mu \nu a}$, i.e. with the antisymmetric 2-tensors in $d=11-p$ dimensions. For the twobrane, the tensor $C_{\mu \nu \rho}$ has kinetic term $H^{-1} F_{4}^{2}$ and extra coupling term $\epsilon_{9} \partial H^{-1} F_{4} F_{4}$, while $C_{\mu a b}$, which has a non-trivial background value, should mix with 'transverse gravitons' $\delta g_{\mu \nu}$. A similar mixing is expected for $C_{\mu \nu \lambda}$ in the fivebrane case.

For the fivebrane there is also another pair of 'mixed' fields (analogous to the system in (A.4), (A.5)): $C_{a b c}$ (scalars in $d=6$ ) and $C_{\mu a b}$ (vectors in $d=6$ ). Their kinetic terms are as in (A.4),

$$
\begin{aligned}
S_{\text {tens. }}= & \frac{1}{2 \kappa_{11}^{2}} \int d^{11} x\left(-\frac{1}{12} H(r)\left[\left(\partial_{i} C_{a b c}\right)^{2}-H(r)\left(\partial_{0} C_{a b c}\right)^{2}\right]\right. \\
& \left.-\frac{3}{4}\left[\left(\partial_{[i} C_{j k] a}\right)^{2}-2 H(r)\left(\partial_{[0} C_{i k] a}\right)^{2}\right]\right)
\end{aligned}
$$


and the mixing term originating from the WZ-term in (2.9) is 11

$$
\epsilon^{\mu \nu \lambda \rho \kappa \sigma} \epsilon^{a b c d e}(\partial C)_{\mu \nu \lambda \rho}(\partial C)_{\kappa \sigma a b} C_{c d e} \sim \epsilon_{a b c d e} \partial_{i} H(\partial C)_{0 i a b} C_{c d e}
$$

The effect of this mixing on the absorption cross-sections remains to be explored.

11 Upon reduction to $d=6$ the relevant interaction term may be written as $W^{\mu \nu} F_{a b \mu \nu} S^{a b}$, where $W^{\mu \nu} \sim \epsilon^{\mu \nu \kappa \lambda \rho \sigma}(\partial C)_{\kappa \lambda \rho \sigma}$ is the dual vector field strength, $F_{a b \mu \nu}$ is the field strength of the vectors $C_{a b \mu}$ and $S^{a b} \sim \epsilon^{a b c d e} C_{c d e}$ are the scalars. In the fivebrane background the vector field strength $W_{\mu \nu}$ has a non-vanishing electric component which produces mixing between the electric component of $F_{a b \mu \nu}$ and the scalars. 


\section{References}

[1] J. Polchinski, Phys. Rev. Lett. 75 (1995) 4724, hep-th/9510017.

[2] A. Strominger and C. Vafa, Phys. Lett. B379 (1996) 99, hep-th/9601029.

[3] C.G. Callan and J.M. Maldacena, Nucl. Phys. B472 (1996) 591, hep-th/9602043.

[4] A. Dhar, G. Mandal and S. R. Wadia, Phys. Lett. B388 (1996) 51, hep-th/9605234.

[5] S.R. Das and S.D. Mathur, Nucl. Phys. B478 (1996) 561, hep-th/9606185; hepth/9607149.

[6] S. Das and S.D. Mathur, hep-th/9607149.

[7] S.S. Gubser and I.R. Klebanov, Nucl. Phys. B482 (1996) 173, hep-th/9608108.

[8] J.M. Maldacena and A. Strominger, hep-th/9609026.

[9] S.S. Gubser and I.R. Klebanov, Phys. Rev. Lett. 77 (1996) 4491, hep-th/9609076.

[10] C.G. Callan, Jr., S.S. Gubser, I.R. Klebanov and A.A. Tseytlin, hep-th/9610172; I.R. Klebanov and M. Krasnitz, hep-th/9612051.

[11] I.R. Klebanov and S.D. Mathur, hep-th/9701187.

[12] S. Hawking and M. Taylor-Robinson, hep-th/9702045.

[13] F. Dowker, D. Kastor and J. Traschen, hep-th/9702109.

[14] E. Witten, Nucl. Phys. B460 (1996) 335.

[15] I.R. Klebanov and L. Thorlacius, Phys. Lett. B371 (1996) 51, hep-th/9510200; S.S. Gubser, A. Hashimoto, I.R. Klebanov and J.M. Maldacena, Nucl. Phys. B472 (1996) 231, hep-th/9601057.

[16] R. Garousi and R. Myers, hep-th/9603194.

[17] A. Hashimoto and I.R. Klebanov, Phys. Lett. B381 (1996) 437, hep-th/9604065.

[18] S.S. Gubser, I.R. Klebanov and A.W. Peet, Phys. Rev. D54 (1996) 3915, hepth/9602135.

[19] I.R. Klebanov and A.A. Tseytlin, Nucl. Phys. B475 (1996) 165, hep-th/9604089.

[20] G. Horowitz and J. Polchinski, hep-th/9612146.

[21] M.J. Duff, H. Lü and C.N. Pope, Phys. Lett. B382 (1996) 73, hep-th/9604052.

[22] H. Lü, S. Mukherji, C. Pope and J. Rahmfeld, hep-th/9604127.

[23] I.R. Klebanov, hep-th/9702076.

[24] M.J. Duff and J.X. Lu, Phys. Lett. B273 (1991) 409.

[25] G.W. Gibbons, G.T. Horowitz and P.K. Townsend, Class. Quant. Grav. 12 (1995) 297, hep-th/9410073; M.J. Duff, G.W. Gibbons and P.K. Townsend, Phys. Lett. B332 (1994) 321.

[26] J.H. Schwarz, Nucl. Phys. B226 (1983) 269.

[27] E. Bergshoeff, C.M. Hull and T. Ortín, Nucl. Phys. B451 (1995) 547, hep-th/9504081.

[28] E. Bergshoeff, H.J. Boonstra and T. Ortín, Phys. Rev. D53 (1996) 7206, hepth/9508091.

[29] G. Horowitz and A. Strominger, Nucl. Phys. B360 (1991) 197. 
[30] M.J. Duff and K.S. Stelle, Phys. Lett. B253 (1991) 113.

[31] R. Güven, Phys. Lett. B276 (1992) 49.

[32] E. Cremmer, B. Julia and J. Scherk, Phys. Lett. B76 (1978) 409.

[33] W.G. Unruh, Phys. Rev. D14 (1976) 3251.

[34] D. Page, Phys. Rev. D13 (1976) 198; Phys. Rev. D14 (1976) 3260.

[35] S. Das, G. Gibbons and S. Mathur, hep-th/9609052.

[36] B. Kol and A. Rajaraman, hep-th/9608126.

[37] J. Maldacena, hep-th/9611125.

[38] S.S. Gubser, November 1996, unpublished notes.

[39] M. Cederwall, A. von Gussich, B.E.W. Nilsson, and A. Westerberg, hep-th/9610148;

M. Cederwall, A. von Gussich, B.E.W. Nilsson, P. Sundell, and A. Westerberg, hepth/9611159.

[40] M. Aganagic, C. Popescu and J.H. Schwarz, hep-th/9612080; E. Bergshoeff and P.K. Townsend, hep-th/9611173.

[41] A. Hashimoto, hep-th/9608127.

[42] R.G. Leigh, Mod. Phys. Lett. A4 (1989) 2767; M. Li, Nucl. Phys. B460 (1996) 351, hep-th/9510135; M. Douglas, hep-th/9512077.

[43] A.A. Tseytlin, Nucl. Phys. B469 (1996) 51, hep-th/9602064.

[44] A. Wasserman, Part III lectures given at Cambridge University, Michaelmas 1995.

[45] J.M. Maldacena and A. Strominger, hep-th/9702015.

[46] S.A. Teukolsky, Astrophys. J. 185 (1973) 635.

[47] E. Bergshoeff, E. Sezgin and P.K. Townsend, Phys. Lett. B189 (1988) 75.

[48] E. Bergshoeff, M. de Roo and T. Ortín, hep-th/9606118; M. Aganagic, J. Park, C. Popescu and J.H. Schwarz, hep-th/9701166; I. Bandos, K. Lechner, A. Nurmagambetov, P. Pasti, D. Sorokin and M. Tonin, hep-th/9701149.

[49] G.W. Gibbons and P.K. Townsend, Phys. Rev. Lett. 71 (1993) 3754; D.M. Kaplan and J. Michelson, Phys. Rev. D53 (1996) 3474, hep-th/9510053.

[50] L. Alvarez-Gaumé and E. Witten, Nucl. Phys. B234 (1983) 269.

[51] M.R. Douglas, hep-th/9604198.

[52] I.R. Klebanov and A.A. Tseytlin, Nucl. Phys. B475 (1996) 179, hep-th/9604166.

[53] V. Balasubramanian and F. Larsen, Nucl. Phys. B478 (1996) 199, hep-th/9604189.

[54] M. Cvetič and D. Youm, Phys. Rev. D53 (1996) 584, hep-th/9507090.

[55] A.A. Tseytlin, hep-th/9609212; hep-th/9702163.

[56] A.A. Tseytlin, Mod. Phys. Lett. A11 (1996) 689, hep-th/9601177.

[57] M. Douglas, J. Polchinski and A. Strominger, hep-th/9703031. 